\title{
A survey of next generation mobile communications research in China
}

\author{
TIAN JinFeng ${ }^{1,2}$, ZHENG XiaoYing $^{2,3}$, HU HongLin $^{1,2^{*}} \&$ YOU XiaoHu ${ }^{1,3}$ \\ ${ }^{1}$ Shanghai Research Center for Wireless Communications (WiCO), Shanghai 200335, China; \\ ${ }^{2}$ Shanghai Institute of Microsystem and Information Technology (SIMIT), Chinese Academy of Sciences (CAS), Shanghai 200050, China; \\ ${ }^{3}$ National Mobile Communications Research Laboratory, Southeast University (SEU), Nanjing 210096, China
}

Received March 25, 2011; accepted June 21, 2011

\begin{abstract}
With the rapid development of wireless communication networks, the fourth generation $(4 \mathrm{G})$ has emerged to move beyond the limitations of the third generation $(3 \mathrm{G})$, with increased bandwidth, enhanced quality of services, and reduced costs of resources. In this paper, we discuss the key technologies of $4 \mathrm{G}$ and focus on surveying the ongoing research in $4 \mathrm{G}$ communications in China. The 4G technologies under investigation include multicarrier transmission, multiple antenna techniques, carrier aggregation, relays, cognitive radio, distributed antenna systems, network convergence, and network self-optimization. These technologies are the building blocks of $4 \mathrm{G}$ and contribute largely to the requirements of International Mobile Telecommunications Advanced (IMT-Advanced). For each technology, its benefits, research topics, and some existing approaches are examined. Finally, we briefly discuss the challenges and future research issues in $4 \mathrm{G}$ systems.
\end{abstract}

multicarrier transmission, multiple antenna techniques, carrier aggregation, relay, cognitive radio, distributed antenna system, network convergence, network self-optimization

Citation: Tian J F, Zheng X Y, Hu H L, et al. A survey of next generation mobile communications research in China. Chinese Sci Bull, 2011, 56: 2875-2888, doi: $10.1007 / \mathrm{s} 11434-011-4658-\mathrm{x}$

The limitations of the third generation (3G) and the rapid growth in user demands has called for more advanced and efficient technologies for new generation systems. The upcoming fourth generation (4G) mobile communication systems are expected to solve the still-remaining problems of $3 \mathrm{G}$ systems and to provide a wide variety of adaptable services for mobile and nomadic users by using integrated heterogeneous network infrastructure. Based on a comprehensive and secure internet protocol (IP) solution, various services, such as voice, video, broadcasting media, and Internet, will be provided to users with high data rates anytime and anywhere. This is also known as the always best connected concept stating that a user can have the best service connection regardless of time and location.

According to the steadily increasing demands and technology developments, wireless communication evolution

\footnotetext{
*Corresponding author (email: hlhu@ieee.org)
}

can be grouped into different generations of networks. Each new generation has brought advances that revolutionized the field of mobile communication. The first generation $(1 \mathrm{G})$, initiating commercial mobile voice services with analog techniques, built the basic structure of mobile communications and solved many fundamental problems such as the multiplexing frequency band, roaming across domains, non-interrupted communication in mobile circumstances, and so on. The second generation $(2 \mathrm{G})$ of wireless technology, which marked the switching of mobile communication technology from analog to digital, offered higher capacity and lower costs for network operators as well as messages and low-rate data services for users, by using a circuit switch infrastructure. Working under 2G's framework, $2.5 \mathrm{G}$ brought the internet into mobile personal communications based on a packet switched radio connection technique. 3G systems, a mixture of packet and circuit-switched networks, are committed to achieving greater network capacity 
through improved spectral efficiency and offer users a wider range of more advanced services such as wide area wireless voice telephony, video calls, and broadband wireless data. Even though $3 \mathrm{G}$ data rates are already reasonably fast, they do not meet the requirements of future high-performance applications. This growing discontent with the limitations of $3 \mathrm{G}$ has led to increasing interest in $4 \mathrm{G}$ networks.

The future 4G infrastructures will encompass all systems from public to private, operator-driven broadband networks to personal areas, and ad hoc networks, using IP as the common protocol. Faster wireless broadband enables "always-on" high-data-rate connections so that streamed audio and video, video messaging, video telephony, mobile TV, and gaming will be constantly at hand. Based on the developing trends in mobile communication, 4G will have increased bandwidth, high data rates (up to $100 \mathrm{Mbit} / \mathrm{s}$ for high mobility and up to $1 \mathrm{Gbit} / \mathrm{s}$ for low mobility), low latency, efficient spectrum use, and low-cost implementations. A $4 \mathrm{G}$ system is intended to become a platform capable of providing more interoperability across multiple communication protocols, and user friendly, innovative, and secure applications. Seamless roaming across different access technologies is provided in $4 \mathrm{G}$ systems. Users are provided a personalized applet, which will allow them to configure a mobile terminal and choose services according to their preferences, with full control over privacy, security risks, and costs. This extraordinary vision for $4 \mathrm{G}$ networks and services is a natural extension of the current development of broadband internet and $3 \mathrm{G}$ mobile networks. With the impressive capabilities of networks and terminals, 4G enhancements promise to bring an entirely new experience, which could potentially lead to growth in other areas such as virtual presence and navigation, tele-medicine and education, tele-geoprocessing, and crisis-management application.

As an agency of the United Nations, the International Telecommunication Union (ITU) coordinates the efforts of government, industry, and the private sector in paving the way for $4 \mathrm{G}$ technologies. To meet the high demand for wireless broadband services by the fast-growing community of mobile users, ITU issued an invitation for radio-access technologies beyond International Mobile Telecommunications 2000 (IMT-2000), which is also referred to as IMTAdvanced. The main official guideline is the IMT-Advanced requirements [1] provided by the ITU's Radiocommunication Sector (ITU-R), which specifies the objectives of the future development of the IMT-Advanced family. The current $4 \mathrm{G}$ development efforts in standardization form the basic stage in the upcoming $4 \mathrm{G}$ process. Recently, two main technologies, the Third Generation Partnership Project (3GPP) Long Term Evolution Advanced (LTE-Advanced), and Worldwide Interoperability for Microwave Access (WiMAX) Release 2, have been recognized as official $4 \mathrm{G}$ technologies. The former is a backwards-compatible enhancement of 3GPP LTE Release 8, while the latter is based on the Institute of Electrical and Electronics Engineers (IEEE) $802.16 \mathrm{~m}$ standard. The specifications of these technologies are now in the final stage of the IMT-Advanced process, which provides for the development in early 2012 of an ITU-R Recommendation specifying the in-depth technical standards for these radio technologies.

The Chinese government has demonstrated strong support for the development of next generation mobile communications by launching the Future Technologies for Universal Radio Environment (FuTURE) project [2] in the 863 program (www.863.gov.cn) of China's Science and Technology Development Plan. The FuTURE Mobile Communications Forum was founded to organize the R\&D activities, accumulate fruitful academic achievements, and accelerate the process of advanced communication systems (www.future-forum.org). Evolution based on time division duplex (TDD) technology has been carried out smoothly and the time diversion, long term evolution, advanced (TD-LTE-A) technology has been successfully approved by ITU as one of the $4 \mathrm{G}$ standards, which will not only promote the industrial development of TD-LTE but also benefit the Chinese mobile communication industry. In 2008, according to the national medium- and long-term plan, the Ministry of Industry and Information Technology of China launched a national key project, the new generation broadband wireless mobile communication networks (www.miit. gov.cn). Many key technologies of future communication systems such as multiple antenna and carrier aggregation technologies have been studied. This project further organizes researchers to focus on the $R \& D$ of communication products, including base stations, terminals and chips, to accelerate the development of the communication industry.

\section{Key technologies}

$4 \mathrm{G}$ has rapidly emerged as one of the most interesting and promising topics in the telecommunication community. Newly advanced technologies are required to implement the impressive features of $4 \mathrm{G}$ systems. Several key $4 \mathrm{G}$ technologies have already been identified and discussed in the literature. In this section we discuss some promising techniques that are likely to have a significant impact on future mobile communication systems.

\subsection{Multicarrier transmission}

The growing demand for multimedia services requires high data rate communications, but this condition is significantly limited by inter symbol interference (ISI) due to the existence of multiple paths. Multicarrier transmission is emerging as a promising technique to combat this problem. As a bandwidth efficient multi-carrier modulation technique, orthogonal frequency division multiplexing (OFDM), which transmits data in parallel using a set of orthogonal carriers 
with harmonic frequency spacing, is considered to be a fundamental technology for future communication systems. It offers high spectral efficiency, low-complexity implementation, and robustness against multi-path fading. With these advantages, the OFDM technique has been adopted by several digital communication standards, such as the European digital audio and video broadcasting standards, wireless local area networks (WLAN), and wireless metropolitan area networks (WMAN). On the other hand, the generalized multi-carrier (GMC) approach establishes a unified description for various multi-carrier as well as single-carrier techniques. Because there is no orthogonal assumption between subcarriers, GMC is suitable for use in a high-mobility environment or where both the Doppler shift and timedelay spread are large.

An OFDM signal exhibits significant amplitude fluctuations over time, which generates a high peak-to-average power ratio (PAPR) due to the nonlinearity of the high power amplifier at the transmitter. The PAPR destroys the orthogonality between the carriers and introduces intermodulation distortion, which lead to high adjacent channel interference and degradation of the bit error rate (BER). The exponential companding scheme proposed in [3] can effectively reduce PAPR for different modulation formats and sub-carrier sizes in OFDM systems, by adjusting the amplitudes of both large and small input signals. Furthermore, this non-linear companding transform approach offers good system performance in terms of PAPR reduction, power spectrum, BER, and phase error. In addition to the companding scheme, partial transmit sequence and selective mapping are two popular PAPR reduction techniques. Both schemes generate multiple representations from the original signal and choose the one exhibiting the best PAPR for transmission. Recently, joint spreading and inverse fast Fourier transforms have been presented to simplify the generation of multiple candidate signals in OFDM-code division multiple access (OFDM-CDMA) systems [4]. Based on a serial peak cancellation mode, the work in [5] proposes an improved peak cancellation scheme to mitigate the interference by an optimized processing order, so as to alleviate the peak re-growth in traditional schemes.

OFDM is a promising technique for broadband wireless communications. However, its performance is sensitive to timing and carrier synchronization errors, resulting in severe performance degradation. To overcome this difficulty, two category approaches, data-aided and non-data-aided, have been proposed to perform timing and frequency synchronizations either jointly or individually. A comprehensive survey can be found in [6]. The data-aided category, using training sequence or pilot symbols, has higher accuracy and lower computation cost, but decreases the data transmission speed. To improve bandwidth efficiency, characteristics such as null subcarriers, constant modulus, and cyclostationarity have also been used to correct synchronization errors in OFDM systems, as non-data-aided approaches.
The non-data-aided category, which relies only on the received symbols, is often desirable for high-data-rate occasions, but is not suitable for acquisition. The sufficient and necessary conditions for null subcarrier allocation that achieves a full estimation range are investigated in [7]. This reference further devises a distributed null subcarrier replacement method for frequency offset estimation. To investigate the effect of time and frequency synchronization errors on system performance, the upper bounds for normalized interference power (NIP) are studied in OFDM systems, which in turn can be used to develop time and frequency synchronization algorithms to achieve a specific NIP value under a given channel condition [8]. In [9], two criteria are used to evaluate the effect of carrier frequency offset on performance degradations in polynomial cancellation coding OFDM systems over additive white Gaussian noise (AWGN) channels.

In addition, OFDM can be combined with other techniques to further improve performance. Many technologies fundamentally based on OFDM, such as vector OFDM (V-OFDM), wide-band OFDM (W-OFDM), flash OFDM (F-OFDM) have shown their distinct advantages in certain applications. Moreover, OFDM modulation can be employed as a multiple access technology, i.e. orthogonal frequency division multiple access (OFDMA). This not only provides additional flexibility for resource allocation (increasing the capacity), but also enables cross-layer optimization of radio link usage, for example cross-layer packet scheduling [10].

The framework of the GMC principle enables a unified description of various modulation schemes, which has attracted much attention in the literature. In [11], Gao et al. present a GMC radio transmission technique for multiple antenna transmission systems, and briefly discuss several key techniques including system design, timeslot structure, pilot design, and channel estimation. An efficient digital implementation of a multicarrier transmission scheme using generalized discrete Fourier transform (DFT) filter banks in multicarrier CDMA systems has been developed in [12]. Based on the filter-bank transform theory, a DFT-spread generalized multi-carrier (DFT-S-GMC) scheme is proposed for uplink transmission of broadband wireless communication [13]. Moreover, a comparison is given of the performance of DFT-S-GMC and DFT spread OFDM (DFT-S-OFDM) schemes in terms of robustness to PAPR and multi-user interference. Additionally, the work in [14] discusses the impact of non-perfect orthogonal prototype filters on the performance of the DFT-S-GMC system. It further presents the single sub-band frequency-domain equalization (FDE) method and analyzes the performance loss caused by FDE-tone discarding.

DFT-S-GMC modulation, as a promising technique for broadband uplink transmission, has been discussed actively in 3GPP standards. To simplify the understanding of the implementation of the DFT-S-GMC technique, the proposal 
in [15] describes the components involved in the user equipment (UE) transmitter in detail, and introduces the key mathematical basis for the DFT-S-GMC transmitter. A comparison of the performance of DFT-S-GMC and DFTS-OFDM in terms of PAPR, synchronization, and BER [16] shows that DFT-S-GMC has low PAPR performance with spreading gains in the frequency-domain, even better than that of DFT-S-OFDM. Moreover, this scheme is more robust against time-frequency synchronization errors and multi-access interferences than DFT-S-OFDM, while the BER performance falls in between the two types of DFTS-OFDM schemes. Ref. [17] further proves that the DFT-SGMC transmission approach has a much smaller phase noise sensitivity than that of the DFT-S-OFDM scheme.

\subsection{Multiple antenna techniques}

The challenge for wireless broadband access lies in providing high speed wireless transmission with good quality of service (QoS) and range capability. The increasing demands for $4 \mathrm{G}$ broadband wireless communications call for application of multiple antennas at both the transmitter and receiver. As one of the most interesting and promising areas of recent innovations in wireless communications, multiple antenna techniques can accomplish multiplexing gain, diversity gain, and antenna gain, thus significantly increasing system capacity, improving link quality, and extending cell coverage. Accordingly, there are three techniques related to multiple antennas gains: spatial multiplexing, spatial diversity, and beamforming. When multiple antennas are used at both ends of the wireless link, these three different methods are often collectively referred to as multiple input multiple output (MIMO) communication. The same sets of antenna configurations of MIMO schemes are targeted in LTE-A and $802.16 \mathrm{~m}$ standards: 2, 4, or 8 transmit antennas and a minimum of 2 receive antennas in the DL, and 1,2 , or 4 transmit antennas in the uplink with a minimum of 2 receive antennas.

Spatial multiplexing boosts the bit rate by transmitting independent information streams (often called layers) on parallel spatial channels associated with the transmit antennas. The first known spatial multiplexing approach, called the Bell Labs layered space-time (BLAST), was invented and prototyped at Bell Labs. To guarantee a certain error performance, channel coding, e.g., horizontal coding, vertical coding, or diagonal coding (a combination of horizontal coding and vertical coding), is often used in conjunction with the spatial multiplexing scheme, with such approaches referred to as H-BLAST, V-BLAST, and D-BLAST, respectively. The V-BLAST architecture has been widely studied in the literature because of its high spectral efficiency and low complexity. An efficient hardware implementation has been developed for a V-BLAST receiver in [18]. Based on the combination of the maximum likelihood (ML) and zero-forcing algorithms, the work in [19] proposes a reduced ML algorithm for a V-BLAST scheme to achieve lower complexity than the ML algorithm while achieving the same performance. The transmit correlation effect on the zero-forcing receiver with successive interference cancellation is investigated for V-BLAST systems in terms of signal-to-noise ratio (SNR) loss and decision error propagation [20]. In [21], the authors analyze the ergodic capacity of spatial multiplexing over spatially correlated rank-1 Ricean fading MIMO channels. By using the statistical channel state information at the transmitter, the work in [22] investigates statistical eigenmode transmission over jointly correlated MIMO channels. It further exploits convex optimization techniques to develop low-complexity power allocation solutions involving only the channel statistics.

In contrast to spatial multiplexing with emphasis on throughput, spatial diversity is designed to combat the detrimental effects in wireless fading channels by transmitting and/or receiving redundant signals representing the same information sequence, so as to reduce the error rate of a system. To generate a redundant signal, space-time coding is used together with a transmit diversity scheme to extract the total available diversity in the MIMO channel, through appropriate construction of the transmitted space-time codewords. For multi-user CDMA systems with different space-time codes, a low-complexity multi-user receiver with linear decoding complexity and good BER performance has been developed over a Rayleigh fading channel [23]. In [24], a distributed space-time-frequency coding (STFC) scheme is proposed for cooperative OFDM systems, which can achieve both spatial and multipath (frequency) diversity. Space-time block coding (STBC), as a wellknown space-time coding technique, achieves a reasonable tradeoff between performance and complexity. For STBC systems with unknown co-channel interference, an oblique projection-based robust linear receiver is proposed to recover the desired STBC encoded signal [25]. Capacity analysis of orthogonal space-time block codes (OSTBCs) systems in multiple input single output (MISO) fading channels with co-channel interference and noise is addressed in [26].

Apart from higher data rates and lower error rates, the available antenna elements can also be used to improve $\mathrm{SNR}$ at the receiver and to suppress co-channel interference (CCI) in a multi-user scenario. Both goals can be realized by means of the beamforming technique. Beamforming adjusts the beam pattern of the transmitted and received signals to enhance the array response in preferred directions. This can be interpreted as linear filtering in the spatial domain. A detailed overview of beamforming is provided in [27]. Recently, an intercarrier interference (ICI) eliminating beamforming scheme has been developed to achieve good symbol error rate (SER) performance for multipath delay profiles, by using a per-tone processing approach [28]. The work in [29] derives a universal upper bound on the average SER (ASER) of transmit beamforming, which is tight in 
uncorrelated and weakly correlated cases, but loose in strongly correlated cases. To take advantage of these multiple antenna techniques, a beamforming scheme employed for array gains or CCI suppression can be combined with spatial multiplexing or spatial diversity techniques to improve the overall performance [30,31].

Using significantly more antennas at enhanced node B (eNB) than those at the terminals calls for the application of multi-user MIMO (MU-MIMO), where the receiving antennas are distributed over multiple users. The base station (BS) may select a subset of users to serve in order to maximize the total throughput of the MU-MIMO system. In [32], an improved norm-based user selection algorithm is presented for MU-MIMO systems with block diagonalization, which selects a subset of users to maximize the total throughput while keeping the complexity low. Two particle swarm optimization scheduling methods are investigated in a downlink MU-MIMO system, which, with different objective functions, are applicable to different capacity and complexity requirements [33]. In the downlink of MUMIMO systems, the CCI and noise are two major impairments. To suppress co-channel interference (CCI) and noise, a channel adaptive power allocation scheme based on signal-to-leakage-plus-noise ratio (SLNR) precoding has been proposed for multi-user MIMO systems, where the value of each user's SLNR is used as the reference for power allocation [34]. This scheme can significantly improve the BER performance with a minor loss in total capacity. With up to four antennas in the uplink of MIMO systems, the complexity and cost required to implement multiple transmitting power amplifiers at the user side are high, even in the case of only two amplifiers. To alleviate these problems, uplink virtual MIMO transmission has been investigated actively in terms of system performance [35] and user paring [36].

Multiple antenna techniques undoubtedly provide large gains with respect to spectrum efficiency. Furthermore, they can increase cell-edge throughput with the help of cooperative transmission. Cooperative multipoint (CoMP) transmission and reception is a framework that enables UE communication with several geographically distributed antenna nodes at the common cooperation area simultaneously, thus serving the UE more efficiently. For multiple nodes working in a coordination transmission mode, a power allocation scheme [37] has been presented to support reliable CoMP transmission. In addition, CoMP comprises a promising spectrum of techniques that still need to be further studied, including channel estimation and feedback, reference signal design, cyclic prefix/OFDM parameters, and backhaul aspects. Moreover, the tradeoff between performance and complexity is still an open question.

\subsection{Carrier aggregation}

Because of the constraints on terminal size and complexity, and the cost limitation of the number of antennas, the transmission performance offered by multi-antenna techniques cannot be continuously increased. To further support wider bandwidth (up to $100 \mathrm{MHz}$ for a single UE unit) while at the same time retaining backward compatibility with legacy systems, carrier aggregation (CA) was introduced by the $3 \mathrm{GPP}$ in its LTE-Advanced standards to aggregate two or more component carriers into one wider channel, despite these spectrum fragments being discontinuous. This aggregation provides extended bandwidth to achieve the desired peak data rates and increase average data rates for users. As one of the key technologies of IMTAdvanced, carrier aggregation has attracted much attention from communication companies such as China Mobile, Nokia, Ericsson, Huawei, and ZTE, amongst others.

In general, CA may be used in three different spectrum scenarios including intra-band contiguous $\mathrm{CA}$, intra-band non-contiguous $\mathrm{CA}$, and inter-band non-contiguous $\mathrm{CA}$. Several typical deployment scenarios, covering both contiguous and non-contiguous carrier aggregation for single and multiple spectrum bands using TDD and frequency division duplex (FDD) schemes, are demonstrated in [38]. Moreover, asymmetric bandwidth for FDD is supported with CA in LTE-A systems to achieve flexible spectrum usage. As for the types of component carriers (CC) deployed at an eNB, three different categories of CCs are defined by 3GPP: backward-compatible carrier, non-backward-compatible carrier, and extension carrier. Backwardcompatible carriers support all of the current LTE features, whereas only LTE-Advanced UEs can access non-backward-compatible carriers. The extension carrier is a part of a component carrier set where at least one of the carriers in the set is a stand-alone-capable carrier. One of the main benefits of the extension carrier is to save control signaling overhead. It is possible to configure all component carriers that are LTE Release 8 compatible, while not all component carriers have to be backward-compatible. An LTEAdvanced terminal with reception and/or transmission capabilities for carrier aggregation can simultaneously receive and/or transmit on multiple component carriers.

The standard research on CA in LTE-Advanced Release 10 is close to the end of the work item phase. In the standard research on $\mathrm{CA}$, the research progress of CA mainly focuses on the following aspects: guard bandwidth between carriers, data stream aggregation, control channels, and uplink power control. Hitherto, most of these topics have already been closed. For contiguous carrier aggregation, the required frequency spacing between the adjacent component carriers should be a multiple of $300 \mathrm{kHz}$ [39]. In principle, aggregation of the component carriers can be done at different layers in the protocol stack. The use of one transport block (in the absence of spatial multiplexing) and one hybrid-ARQ entity for each scheduled component carrier has finally been determined as the baseline for data aggregation schemes in LTE-A systems [40]. In terms of control channel design, the basic framework for control signaling with CA 
has already been approved. The design principles for downlink control signaling and uplink control signaling can be found in the specification of TR36.814 [43]. The standardization of uplink power control in the case of CA focuses mainly on the power limitation problem, power headroom reporting, and TPC command transmission, conclusions of which have almost been finalized.

Research on CA technology has been very active in recent years. The work in [41] reviews the detailed proposals of the key aspects in CA standardization at the time of the investigation. The performance gain of carrier aggregation technology over independent carrier schemes is evaluated based on different scheduler structures and different traffic models [42]. The design of the scheduling scheme is one of the key aspects in the performance of the LTE-Advanced system using CA. The study in [43] provides two user allocation methods based on a traffic load balancing rule in the same frequency band, while a multi-band scheduling scheme over two frequency bands is explored in [44]. Separate random user scheduling and joint user scheduling are analyzed in terms of QoS performance in [45]. To mitigate inter-cell interference, the authors in [46] modeled carrier assignment in downlink CoMP (coordinated multi-point transmission/reception) with $\mathrm{CA}$ as a graph coloring problem to maximize the performance of both the network and individual user. In [47], a flexible carrier aggregation scheme with autonomous carrier management is recommended for home base stations.

\subsection{Relay}

The envisaged high bandwidth for IMT-Advanced systems may reduce the available spectral power density, and furthermore, wide spectrum is only available at higher frequency bands implying higher attenuation especially for indoor broadband services. To combat these effects and to improve the throughput, transmitting nodes need to be placed closer to the users to achieve very high user data rates. This configuration is usually called a relay or multihop network. The relay transmission helps to forward the information to destinations, and can be seen as a kind of collaborative communication. Relay networking has been studied in ad hoc networks, peer-to-peer networks, and broadband cellular systems. A denser infrastructure can be achieved by deploying relay nodes for coverage extension and throughput enhancement. Additionally, relay based systems typically include the advantages of low cost and reduced power consumption.

With the development of communication systems, relay technologies have been studied actively in the standardization process of next generation mobile communication systems, such as 3GPP LTE-Advanced and IEEE $802.16 \mathrm{~m}$. The standards have defined two types of relay nodes (RN). 3GPP uses Type 1 and Type 2 RNs in LTE-Advanced systems, while IEEE $802.16 \mathrm{~m}$ uses non transparent and transparent RNs. An introduction to and comparison of the different relay types in LTE-Advanced and WiMAX standards can be found in [48]. Regarding transmission schemes for RNs, many methods have been developed over the last decade. Amplify-and-forward relays amplify the received signal and forward it to the UE (or eNB), while decode-andforward relays decode the signal and then re-encode it for transmission. Besides the two typical transmission schemes, there are several hybrid types of transmission, such as the compress-and-forward and the demodulate-and-forward. With respect to the spectrum usage of the relay node, communication between the RN and the eNB can be effected in two ways: in-band and out-band. In in-band mode, the eNBrelay link shares the same band with the relay-UE links within the donor cell, while in out-band mode, a different band is used.

Evaluation of the cost and performance of relayenhanced networks has already started. The study in [49] analyzes the techno-economic viability of relay solutions based on fairness allocation criteria, while an analysis of the performance of dual-hop communication systems with fixed gain amplify-and-forward relay in terms of outage probability and ASER over Nakagami- $m$ fading channels is given in [50]. In [51], the service connectivity and user access probabilities are investigated in infrastructure-based vehicular relay networks. Specifically, the impact of the density and the coverage ranges of the two types of nodes (BS and RN) on these important performance metrics is studied in this work. Performance of cooperative networks with random decode-and-forward relays is analyzed in [52], where the number of relays is a binomial random variable. This shows that both diversity and coding gain are affected by the RN distribution.

Though extensive research has been carried out on feasibility analysis of the use of RNs in different relay network scenarios, integrating relays into a system involves several functionalities that must be taken into account. The authors in [53] presented a new preamble structure and corresponding timing and frequency synchronization algorithms for cooperative relay systems. In [54], based on a simple semi-orthogonal pilot structure, a low complexity channel estimation approach is proposed to obtain both performance and computational complexity advantages in bi-directional relaying networks, which also saves the frequency spectrum resource. Additionally, resource allocation policies in relay networks require the design of relay selection [55], access strategies [56], and power allocation [57], amongst others, under certain QoS constraints. Furthermore, the introduction of relay stations has made the handover process more complicated, requiring more signaling overhead and processing delay. The fast handover scheme for IEEE 802.16-based relay networks in [58] determines the cross-subnet handover in advance through the interaction of MAC layer messages, which can reduce the handover signaling cost and decrease handover delay. 
In addition, the performance of RNs can be further improved by using combined techniques. Relaying has been combined with multiple antennas in MIMO relay networks to achieve the improved performance in terms of outage probability and symbol error rate (SER) [59,60]. For a MIMO-OFDM relaying communication system, an optimal power allocation algorithm is introduced in [61] to improve the system capacity and enhance the transmission ability. Under the assumption of known channel state information, two optimal relay schemes with local relay power limits are proposed in [62] to maximize the information rate for two-hop MIMO relay networks, using the normalized and Lagrangian algorithms, respectively. A cross-layer communication strategy between the physical layer and the medium access control (MAC), which can achieve improved throughput and delay performance, has been designed using the Jackson queuing model for multiple relay cooperative networks [63]. The work in [64] develops a cross-layer analytical model to characterize the multi-hop delay performance in a wireless multi-hop environment. In practice, relay cooperation can be introduced into frameworks in many ways with each approach implementing a different tradeoff of power, bandwidth, processing, and economic resources.

\subsection{Cognitive radio}

The limited spectrum resources and inefficiency in the spectrum usage necessitate cognitive radio (CR) techniques to exploit the existing wireless spectrum in an opportunistic manner. A CR is an autonomous wireless unit that can sense its surrounding environment and dynamically adapt its operating characteristics (i.e., frequency band and waveforms) to improve its performance without interfering with licensed users. This maximizes the utilization efficiency of the limited spectrum resources and enables the accommodation of an increasing number of services in wireless networks.

The recent progress in CR research has benefitted from multidisciplinary efforts, e.g., software defined radio (SDR), Bayesian signal processing, game theory, and cross-layer protocol design. SDR is the basic enabling technology for cognitive wireless networks, which implements the radio functionality as software modules running on a generic hardware platform. This programmable technology allows new wireless features and capabilities to be added or the existing radio systems to be reconfigured without requiring extra hardware. As $4 \mathrm{G}$ devices will constitute the entire collection of wireless standards, SDR technology appears to be a cost-efficient solution for implementing several access approaches with different software modules in one terminal. Additionally, they are appealing in overcoming power, size, and compatibility limitations of user equipment.

Cognitive radio, built on a software radio platform, is a context-aware intelligent radio potentially capable of autonomous reconfiguration by learning from and adapting to the communication environment. This adaptive ability can be realized through spectrum management functions in CR networks. The cognitive capabilities of the network devices enable unlicensed users to detect spectrum white space, select the best frequency bands, coordinate spectrum access with other users, and vacate the frequency when current channel conditions become worse or a licensed user appears. These functionalities are also known as spectrum sensing, spectrum selection, spectrum sharing, and spectrum mobility, respectively. In particular, spectrum sensing and spectrum sharing have been investigated widely because of their key roles in CR networks.

Spectrum sensing, providing awareness of spectrum utilization, is the key to efficient spectrum allocation in CR networks. This awareness is often studied at the physical layer and at the MAC layer. Physical layer spectrum sensing, as surveyed in [65], typically focuses on the detection of instantaneous licensed user signals using local spectrum sensing. The typical local spectrum sensing methods include three broad categories: energy detection, matched filtering, and feature detection. Features based on cyclostationarity, eigenvalue, and wavelet analyses have been presented for sensing licensed users using statistical analysis and signal processing techniques. To cope with noise uncertainty, fading, and shadowing, cooperative sensing, as a kind of MAC layer sensing approach, is a natural solution to enhance the detection performance. Recently, the work in [66] proposed a multiple-user cooperative spectrum sensing scheme to achieve spatial diversity gains for cognitive radio networks, where only the relay with the best channel condition is utilized to cooperatively detect the primary user. Certain issues that can piggyback on the cooperation scheme, e.g. false message attack [67] and reporting errors induced by fading channels [68], are also addressed in the literature. In addition, MAC layer spectrum sensing has been investigated to maximize spectrum efficiency and maintain CR user connections, with the aid of traffic prediction [69] and channel state transition probability [70].

Because unlicensed users may share the spectrum resources with licensed users as well as other unlicensed users, efficient dynamic spectrum sharing schemes are critical to achieve high spectrum efficiency. Numerous research efforts have been directed towards the development of novel spectrum sharing technologies. Based on fairness criteria, the authors in [71] have developed a joint channel and power allocation scheme with lower power consumption for centralized cognitive networks. In [72], the Lyapunov optimization method is employed to maximize the utilization efficiency of the idle channels of licensed users for CR networks, using a greedy selection mechanism. A spectrum sharing method based on the Frobenius norm has been proposed for MIMO CR networks to maximize the throughput of CR users as well as guarantee the quality of service of licensed users [73]. Under both the average transmitted and received power constraints, the work in [74] studies the er- 
godic capacity of spectrum sharing systems in fading channels.

Additionally, other topics are presented to facilitate basic spectrum management functionalities in cognitive radio networks, involving common control channel [75], interference management [76], and power control [77], amongst others. As the performance of CR networking functionalities directly depends on the properties of the spectrum band in use, a comprehensive solution, jointly considering beamforming, scheduling and power allocation, has been proposed for CR network downlinks to achieve high sum-rate throughput with tolerable interference [78]. In addition to cooperation across multiple layers in CR networks, applying user cooperation to the cognitive radio, such as relay transmission, provides reliability and increased capacity as well as efficient spectrum utilization. The outage probability minimization for cognitive relay networks is investigated in [79]. Along with cooperative networking, cognitive networking shows potential for highly efficient spectrum usage and spectrum sharing, which leads to increased channel capacity.

\subsection{Distributed antenna system}

The target of future wireless systems is to provide high data rates to a large number of users, which requires improved system capacity. To meet the capacity demand, distributed antenna systems (DAS) have become the mainstream network architecture for $3 \mathrm{G}$ and $4 \mathrm{G}$ communication systems, enabling operators to offer better coverage and to boost network capacity via mini-networks of small nodes, rather than building new towers or acquiring a new spectrum. A DAS is a new kind of network in which multiple antennas are geographically separated from each other, but connected to a common source. This splits the transmitted power among several spatially separated antenna elements so as to provide coverage over the same area as a single antenna, but with reduced total power and improved reliability. DAS technologies can be used for indoor locations-mainly to boost signal coverage in large office buildings or shopping centers-as well as for outdoor purposes.

As a promising wireless network structure, the DAS has attracted many investigations on its application in future wireless communication systems. The work in [80] provides a comprehensive technical guide that covers the fundamental concepts, effective protocols, performance analysis and open issues of the DAS. In [81], the authors investigated the power coverage and fading characteristics of a DAS using measured data in an indoor office scenario. It is inferred that the DAS system can provide more uniform power coverage, higher energy efficiency, and better communication performance, compared with conventional centralized antenna systems. As far as the capacity analysis of a DAS is concerned, many studies have focused on a downlink DAS due to the dominant downlink traffic demand. A good approxi- mation of the ergodic downlink capacity of a DAS with random antenna layout has been designed based on random matrix theory [82]. Additionally, by employing two-step approximations, an approximate analytical expression of the downlink channel capacity has been developed in [83] for a DAS over shadowing Nakagami fading channels.

In a DAS, the distributed antenna transmission approach is a key technology in achieving performance improvement in terms of capacity and SER. Based on the received SNR from each distributed antenna port and the required QoS of the users, the antenna transmission mode is adaptively selected for each mobile station in the distributed wireless communication system, thereby increasing the efficiency and flexibility of the radio resource management (RRM) in the DAS [84]. To optimize the downlink channel capacity of the DAS, a capacity based remote antenna unit (RAU) selection scheme, which combines optimal capacity RAU selection and power allocation, is proposed in [85] for downlink transmission in cellular and Manhattan scenarios. In [86], an adaptive distributed transmit antenna selection scheme is proposed to minimize the SER in a limited feedback beamforming DAS that can adaptively adjust the number of distributed transmit antenna according to large scale fading.

In addition, there are many other issues that need to be considered when applying a DAS to practical wireless communication systems. Considering the composite channel including large-scale and small-scale fading, a downlink power allocation method for a DAS with random antenna layout is investigated in [87]. Specifically, this study proposes a sub-optimal power allocation scheme with low complexity, where the system capacity is shown to be quite close to the optimal one obtained by numerical optimization. By introducing a sectorized distributed antenna structure, a practical beam switching algorithm with optimal power allocation is presented in the DAS to achieve significant gains over SINR and capacity [88]. To maximize the cell averaged ergodic capacity, the squared distance criterion in [89] renders codebook design algorithms in vector quantization applicable to antenna location design. In [90], a parallel proportional fair scheduling scheme is introduced for a DAS to achieve a tradeoff between average throughput and fairness.

Combined with the advances in MIMO techniques, a DAS with multiple antennas at each remote radio unit can utilize the distributed antennas coordinately to achieve additional diversity as well as spatial multiplexing gains. This is usually referred to as distributed MIMO (D-MIMO) or cooperative DAS. An introduction to the concept and key technologies of cooperative DAS systems can be found in [91], which shows that a DAS with a cooperative transmission scheme can provide very promising performance enhancements in capacity and transmit power efficiency. Based on the ML algorithm, the authors in [92] investigated the carrier frequency offsets in MIMO systems with distrib- 
uted transmit antennas, where the received antennas were assumed to be centralized. Spectrum efficiency, as a significant performance metric, is investigated analytically in closed-form for D-MIMO cellular systems under a composite fading channel [93]. For multi-user DAS with multi-antenna array scenarios, the work in [94] analyzes and compares four downlink transmission methods. Of these transmission schemes, the intra block diagonalization method is proven to be a good tradeoff that achieves high capacity with relatively low complexity when considering power constraints. The impact of antenna correlation on power allocation in D-MIMO systems is addressed in [95], which also proposes a low-complexity port selection and power allocation scheme based on large scale fading and antenna correlation.

\subsection{Network convergence}

Nowadays, communication systems are highly heterogeneous and consist of multiple networks, including fixed-line networks (DSL, cable modems, LANs), cellular networks (macro, micro, pico and femto cells), WLAN, WMAN, and satellite systems. The existence and popularity of heterogeneous technologies has created the need for integration and convergence over a common all-IP based platform. This will enable potential users to access different types of communication services (possibly multiple services) anytime and anywhere, thus resulting in increased bandwidth, internet accessibility, coverage area, and user services. In addition, the unified framework supports the expansion of technological capacity for further scientific progress and leads to more competition in individual markets for each of these services.

The need for pervasive and ubiquitous networking requires integration of various wireless solutions into a single platform. The integration focuses on offering seamless interoperability of different types of wireless networks with a wireline backbone. The concept of interoperability requires (user transparent) reconfigurability and cooperation in various communications systems paving the road towards the 4G paradigm. Reconfigurable interoperability can be done at the network level, the user level or both, providing reliable services and allowing user seamless and transparent service management. The seamless handover decision often depends on parameters such as preferences of users and network conditions, with the aim of offering the best connected services always.

The current telecommunication environment is divided into wireless, fixed line, telecommunication, and broadcasting, causing inconvenience to the users. Convergence of the multiple types of networks into a single network is on the horizon, and this has clear advantages in terms of bandwidth, power consumption, spectrum usage, and a mix of heterogeneous services [96]. Based on a converged platform, both users and providers will benefit from improved quality and less complexity at an affordable cost and with single billing. The convergence technologies cover multiple facets: different networks (e.g. fixed-mobile, three-screen convergence of telecom, internet, and broadcasting), different traffic types (e.g. voice, multimedia, and data), different technologies (e.g. computers, consumer electronics, and communication technology), different media (e.g. broadcast, satellite, cellular), different services, and so on.

Integration and convergence of all possible different networks are achieved using IP technology. IP provides independence from the underlying networks and is compatible with the actual radio access technology. The compatibility of various radio protocols means unimpeded innovation all round, accompanied by low cost. As the free address pool of IP version 4 (IPv4) used in current systems is becoming exhausted, IP version 6 (IPv6) has been adopted in $4 \mathrm{G}$ networks to replace IPv4 and this enables high-performance scalable networks. It is essentially a catalyst to inspire innovation in different areas, especially in access infrastructures, home networks, and user applications. The most relevant 4G IP extension is the mobile IP protocol [97], which is considered to be the major player in integration between wireless and wireline network platforms.

With the irreversible trend of convergence and cooperation among heterogeneous networks, some issues caused by heterogeneity, for example QoS, RRM and reconfiguration, have been actively addressed in the literature. Comprehensive coverage of QoS issues in heterogeneous network environments is studied in detail in [98]. An efficient RRM scheme is required to manage mobile device resources in heterogeneous environments. The RRM should fit into the overall picture of heterogeneous network management for next generation networks, which includes techniques such as access management, mobility management, secure management, power management, location management, and so on [99]. The work in [100] investigates the resource reconfiguration model of substrate physical networks and provides a service oriented network architecture as a network convergence primitive.

\subsection{Network self-optimization}

Self-optimization techniques are of vital interest to next generation mobile networks, where the network coverage, capacity and service quality experienced by users are expected to be enhanced, while the capital and operational expenditure should be further reduced. The importance of self-optimization has been recognized by the standardization body 3GPP, which has introduced self-optimization mechanisms in its technical report TR36.902 [101]. Several projects, such as SOCRATES (www.fp7-socrates.eu) and Monotas (www.macltd.com), have been conducted to investigate self-optimization techniques. In [102], Hu et al. introduce self-configuration and self-optimization techniques for LTE networks. In [103], Feng et al. consider cooperation 
among heterogeneous networks assisted by a cognitive pilot channel. Heterogeneous network self-optimization algorithms have been proposed to solve the adaptation problem in reconfigurable systems, to reduce the system blocking rate, and to improve network revenue. Self-optimization techniques include but are not limited to coverage and capacity optimization, mobility robustness optimization, and mobility load balancing optimization.

Capacity optimization is an important issue for future communication networks to deliver services at much higher data rates compared to previous generations. Because there is a tradeoff between network coverage and capacity, the coverage area must be considered along with capacity optimization during network planning. 3GPP TR 36.902 introduced a coverage and capacity optimization use case, in which continuous coverage should be guaranteed together with optimized capacity and coverage optimization has a higher priority than capacity optimization. In [104], the author analyzes the optimization space of antenna parameters and discusses vertical sectorization as a capacity optimization approach. This study further adopts an adaptive antenna system to optimize coverage and capacity.

In cellular networks, unnecessary handovers, handover failures, and radio link failures caused by improper handover parameter settings result in wasted system resources and deteriorated user experiences. The SOCRATES project studied how to tune the handover parameters of a LTE base station to improve the network performance in terms of call dropping ratio, handover failure ratio, and ping-pong handover ratio. In this investigation, by observing the handover performance indicators, the hysteresis and time-to-trigger are tuned accordingly if any of the performance indicators overshoots the threshold. Finally, the best hysteresis and time-to-trigger combination is approached gradually [105]. In [106], the handover parameters (i.e. time-to-trigger, measurement interval, and hysteresis) are adjusted by comparing the number of ping-pong handovers and the number of handovers performed in a measurement interval. To further improve the handover performance, an adaptive layer- 3 filter based on the user's velocity is proposed in this paper. The authors in [107] consider enhancing fast handovers for mobile IPv6 by integrating a candidate access router discovery mechanism and specifying layer-2 triggers. Furthermore, different handover processes are performed for different service types according to the QoS requirements. The overall scheme could improve handover latency. In [108], an intra-domain mobility management protocol is proposed, which adopts a layer-2 fast handover trigger, an edge-cells controller, and the pre-registration method to improve the intra-domain mobility in heterogeneous wireless networks.

The random arrival of mobile users and the resulting unbalanced cell load make efficient resource utilization at reduced system cost a critical challenge for future mobile networks. The SOCRATES project developed a self-opti- mizing load balancing algorithm in an LTE based mobile communication system [109]. In this approach, the cell specific handover offset is modified gradually based on cell load information to force mobile users to handover from overloaded cells to under-loaded cells. The design principal of the algorithm is to find the optimum offset that allows the maximum number of users to change cells without any handover requests being rejected by the target cell. The work in [102] presents a handover penalized load balancing algorithm, which has performance bounds on both the average system delay and the average number of handovers that have taken place. Wang et al. formulated the load balancing problem as a network-wide utility maximization problem and transformed it into an integer optimization problem. A practical suboptimal algorithm has been proposed, which achieves high network throughput at the cost of a few more handovers [110].

\section{Challenges and further research areas}

The 4G system, aimed at providing a set of services anytime and anywhere, poses a number of serious challenges for wireless communications. Various service providers have their own protocols, which may be incompatible with each other as well as with user devices. Too many divergent approaches defined in 4G may further complicate the process of selecting the most appropriate technology and may affect user experience, thus calling for a global standardized approach. In addition, there are many challenges from the technique perspective. The requirements of higher capacity, high rate, and low transfer delay time demand advanced techniques at the physical layer. Coping with new and heterogeneous system architectures, such as mesh networks, multi-hop networks, peer-to-peer communication and multi-standard networks will be a tough enough task. Supporting QoS in $4 \mathrm{G}$ networks will be a major challenge. QoS modeling and signaling are crucial factors in a system that integrates heterogeneous networks. Inter-network (vertical) and intra-network (horizontal) handover connectivity is fundamental to the provision of temporally and spatially seamless services. Additionally, resource allocation, interference mitigation, and security are by no means straightforward in heterogeneous environments. Even with the mature $4 \mathrm{G}$ technologies, $3 \mathrm{G}$ technologies still exist in the market. As such, there is a marketing challenge to develop $4 \mathrm{G}$ because of the continued existence of $3 \mathrm{G}$.

To stabilize and develop $4 \mathrm{G}$ into a sustainable product, some further research areas are considered important. Because achieving high spectral efficiency and handling high frequency-selectivity are major technical challenges, it is crucial to incorporate the recent technical advances in the physical layer into future wireless systems. In this point, an investigation of leading technological advances and future trends in the physical layer enabling truly ubiquitous 
broadband wireless capabilities will be conducted. Topics for advanced physical layer technologies may include new codec and modulation schemes, joint channel estimation, multiple-access techniques, ultra wideband systems and hardware design, amongst others. As mentioned before, 4G communication systems provide both opportunity and challenges to RRM and utilization. Research on RRM algorithms, including access control, resource allocation, load control, and handover control, is still high priority, considering the comprehensive factors in an intelligent and integrated heterogeneous network. Interference induced by complex network environments also impedes the implementation of $4 \mathrm{G}$ systems. Mitigating interference will be an important topic under new deployment circumstances. Because most of the network protocols already have some inherent collaboration, cooperation in multiple forms such as different networks, different nodes, different layers and different technologies, will remain a promising solution in the future.

In addition, green communication, which is dedicated to improving energy efficiency, has become an active research topic recently. The fundamental drivers for green communication are the need to reduce global $\mathrm{CO}_{2}$ emissions and the savings in capital and operational expenditure through reduced energy consumption. The work in [111] aims to save power consumption of user equipment by developing a dynamic discontinuous reception scheme to adjust the inactivity timer and the sleep period of UE receivers. In [112], the authors discuss communication networks from an energy-efficiency point of view and summarize a series of energy consumption models as well as general approaches for developing these models. In 2009, the HuangShan Symposium on "Green Wireless Technology and Systems" was held to bring together academic and industrial researchers to discuss energy-efficient communications [113]. In the symposium, cooperative distributed MIMO systems, content aware soft real time transmission design, and cognitive techniques were discussed and proposed as efficient energy saving techniques. The symposium also discussed the transition from achieving the highest transmitting data rate to seeking optimal power efficiency for future communication networks. Nevertheless, research in green communication is still in its infancy and is far from being adequately explored.

With the pervasiveness of computer networks and mobile communications, the Internet of Things (IoT) has been envisioned as a forthcoming wave in the world information industry and has attracted much interest in academia and industry. The IoT is a network of Internet-enabled objects, where the objects are endowed with the ability to identify themselves, communicate with other objects, and possibly compute. Hitherto, a broad scope of research in this area has been carried out in a variety of projects by different research organizations. The enabling building blocks for the development of the IoT include machine to machine communication, microcontrollers, radio frequency identification (RFID) technologies, sensors, location technology, and so on. Efforts towards standardization have focused on several principal areas: RFID frequency, protocols of communication between readers and tags, and data formats placed on tags and labels. Apart from the technical aspects, the economic perspective of possible applications and quantifiable benefits are additional research tasks. In China, several research institutes have been involved in a far-reaching project with strong support from the Chinese government since 2006, to investigate this emerging research field. It is believed that in the near future the achievement of the vision of "connectivity for anything (including anyone) anytime and anywhere" should depend on cross-discipline and cooperative efforts in related fields.

The concept of being connected anytime, anywhere, via any type of device will require the dreaded "big fat pipe", which places enormous pressure on service providers. To alleviate the heavy computing burden in 4G systems, cloud computing, which provides computational resources on demand via a computer network, may be applied in $4 \mathrm{G}$ services. This new paradigm has been receiving much attention from the research community and industry in China. The Chinese State Council has designated cloud computing as a strategic technology in its 12th Five-Year Plan. Leading research institutes and enterprises in China's electronic information field are making progress on multiple facets of cloud computing, including core technologies, application solutions, and service models. The enterprises, such as China Mobile and China Telecom, have shown great enthusiasm in services based on cloud computing. It can be predicted with a great deal of certainty that the combination of cloud computing and $4 \mathrm{G}$ will be a trend in future networks.

\section{Conclusions}

This paper has provided an overall vision of $4 \mathrm{G}$ communication systems and has addressed the research activities and achievements of Chinese scholars in the progress of $4 \mathrm{G}$. $4 \mathrm{G}$ wireless networks provide a wide variety of services and enable more efficient, scalable, and reliable wireless services. Spectral efficiency can be achieved by OFDM and cognitive radio technologies, while multiple antenna techniques, distributed antenna systems, and carrier aggregation focus on capacity enhancement and high data rates. The enhanced coverage and higher cell edge data rate requirements of $4 \mathrm{G}$ systems could be met by relaying technology. Network convergence necessitates interoperability across communication protocols in $4 \mathrm{G}$ networks, while network self-optimization contributes to overall performance improvements and accelerates the introduction and deployment of new wireless services. Chinese scholars have made great contributions to these key technologies in $4 \mathrm{G}$ systems. Some research issues of these technologies are still open and require further investigation. We believe that future 
research will overcome the difficulties, integrate newly developed services into $4 \mathrm{G}$ networks, and make them available to anyone or anything, anytime and anywhere.

This work was partially supported by the National Natural Science Foundation of China (61002017 and 61072076), the Shanghai Rising-Star Program, the Shanghai Natural Science Foundation (09ZR1430500), and the Open Research Fund of the National Mobile Communications Research Laboratory, Southeast University, China.

1 ITU-R Rec M.2134. Requirements related to technical performance for IMT-Advanced radio interface(s). 2008

2 You X H, Chen G A, Chen M, et al. The FuTURE Project in China. IEEE Commun Mag, 2005, 43: 70-75

3 Jiang T, Yang Y, Song Y H. Exponential companding technique for PAPR reduction in OFDM systems. IEEE Trans Broadcast, 2005, 51: 244-248

4 Ren Z Y, Zhang H L, Guo K. A novel method for the improvement of power efficiency in high peak-to-average-power ratio communication systems. Sci China Inf Sci, 2010, 53: 1697-1702

5 Dan L L, Xiao Y, Ni W, et al. Improved peak cancellation for PAPR reduction in OFDM systems. IEICE Trans Commun, 2010, E93.B: 198-202

6 Ai B, Yang Z X, Pan C Y, et al. On the synchronization techniques for wireless OFDM systems. IEEE Trans Broadcast, 2006, 52: 236-244

7 Shu F, Berber S, Wang D M, et al. ML integer frequency offset estimation for OFDM systems with null subcarriers: Estimation range and pilot design. Sci China Inf Sci, 2010, 53: 2567-2575

8 Shu F, Cheng S X, Lee J, et al. Analysis of time and frequency synchronization error for wireless systems using OFDM. Sci China Ser F-Inf Sci, 2005, 48: 379-396

9 Zhou P, Zhao C M, Shi Z H, et al. Performance evaluation for PCC-OFDM systems impaired by carrier frequency offset over AWGN channels. Sci China Ser F-Inf Sci, 2008, 51: 320-336

10 Wang J B, Chen H M, Chen M, et al. Cross-layer packet scheduling for downlink multiuser OFDM systems. Sci China Ser F-Inf Sci, 2009, 52: 2369-2377

11 Gao X Q, You X H, Jiang B, et al. Generalized multi-carrier transmission technique for beyond $3 \mathrm{G}$ mobile communications. In: Proceedings of the 16th IEEE International Symposium on Personal, Indoor and Mobile Radio Communications, 2005 Sept 11-15, Berlin. Washington DC: IEEE, 2005. 972-976

12 Gao X Q, You X H, Sheng B, et al. An efficient digital implementation of multicarrier CDMA system based on generalized DFT filter banks. IEEE J Sel Area Commun, 2006, 24: 1189-1198

13 Zhang X D, Li M Q, Hu H L, et al. DFT spread generalized multi-carrier scheme for broadband mobile communications. In: Proceedings of the 17th IEEE International Symposium on Personal, Indoor and Mobile Radio Communications, 2006 Sept 11-14, Helsiniki. Washington DC: IEEE, 2006. 1-5

14 Li M Q, Zhang X D. Performance analysis of DFT spread generalized multi-carrier systems. Sci China Ser F-Inf Sci, 2009, 52: 23852396

15 3GPP R1-051134, SHRCWC \& RITT. On the implementation of DFT-S-GMC. TSG-RAN WG1 Meeting \#42bis, 2005

16 3GPP R1-051135, SHRCWC \& RITT. Performance comparison between DFT-S-GMC and DFT-S-OFDM. TSG-RAN WG1 Meeting \#42bis, 2005

17 3GPP R1-051385, SHRCWC. Further simulation results of DFT-SGMC in comparison with DFT-S-OFDM, TSG-RAN WG1 Meeting \#43, 2005

18 Wang Q, Tao X F, Zhang P, et al. Low complexity hardware implementation of V-BLAST receiver. In: Proceedings of the 67th IEEE Vehicular Technology Conference, 2008 May 11-14, Marina Bay. Washington DC: IEEE, 2008. 1355-1359
19 Pan W, Jiang Z J, Du Z F, et al. Analysis of a reduced-ML algorithm in BLAST. Sci China Ser F-Inf Sci, 2008, 51: 2094-2100

20 Guo H Y, Zhou Z G, Hu H L. Transmit correlation effect on SIC-ZF receiver for V-BLAST system. Sci China Ser F-Inf Sci, 2009, 52: 2290-2295

21 Jin S, Gao X Q, You X H. On the ergodic capacity of rank-1 Ricean-fading MIMO channels. IEEE Trans Inf Theory, 2007, 53: 502-517

22 Gao X Q, Jiang B, Li X. Statistical eigenmode transmission over jointly correlated MIMO channels. IEEE Trans Inf Theory, 2009, 55: 3735-3750

23 Yu X B, Dong T, Bi G G. Performance of multiuser CDMA system with space-time block coding in MIMO Rayleigh fading channels. Sci China Ser F-Inf Sci, 2009, 52: 2296-2307

24 Yang W W, Cai Y M, Wang L. Distributed space-time-frequency coding for cooperative OFDM systems. Sci China Ser F-Inf Sci, 2009, 52: 2424-2432

25 Fan J C, Yin Q Y, Wang W J. Robust linear receivers for STBC systems with unknown co-channel interference. Sci China Ser F-Inf Sci, 2009, 52: 1067-1074

26 Yue D W, Wang Q. Capacity of orthogonal space-time block codes in MISO fading channels with co-channel interference and noise. Sci China Ser F-Inf Sci, 2009, 52: 1697-1703

27 Van Veen B D, Buckley K M. Beamforming: A versatile approach to spatial filtering. IEEE Signal Proc Mag, 1998, 5: 4-24

28 Zhang Y, Li J D, Pang L H. Intercarrier interference alleviation beamforming for OFDM with space diversity. J Electron (China), 2010, 27: 321-327

29 Zhu P C, Tang L, Wang Y, et al. An upper bound on the SER of transmit beamforming in correlated Rayleigh fading. IEEE Trans Commun, 2010, 58: 457-462

30 Shu F, Li L H, Tao X F, et al. A spatial multiplexing MIMO scheme with beamforming for downlink transmission. In: Proceedings of the 66th IEEE Vehicular Technology Conference, 2007 Sept 30-Oct 3, Baltimore. Washington DC: IEEE, 2007. 700-704

31 Lin M, Li M, Yang L X, et al. Combined adaptive beamforming with space-time block coding for multi-antenna communications. Sci China Ser F-Inf Sci, 2008, 51: 2062-2073

32 Zhu Y T, Tang Z H, Zhu J K. An improved norm-based user selection algorithm for multiuser MIMO systems with block diagonalization. In: Proceedings of the 66th IEEE Vehicular Technology Conference, 2007 Sept 30-Oct 3, Baltimore. Washington DC: IEEE, 2007. 601-605

33 Hei Y Q, Li X H, Yi K C, et al. Novel scheduling strategy for downlink multiuser MIMO system: Particle swarm optimization. Sci China Ser F-Inf Sci, 2009, 52: 2279-2289

34 Wang J, Wang X T, Guo Y L, et al. A channel adaptive power allocation scheme based on SLNR precoding for multiuser MIMO systems. In: Proceedings of the 72nd IEEE Vehicular Technology Conference, 2010 Sept 6-9, Ottawa. Washington DC: IEEE, 2010. 1-4

35 3GPP R1-051422 Nortel. UL virtual MIMO system level performance evaluation for E-UTRA. TSG-RAN1 WG1 \#43, 2005

36 Chen X, Hu H L, Wang H F, et al. Double proportional fair user pairing algorithm for uplink virtual MIMO systems. IEEE Trans Wirel Commun, 2008, 7: 2425-2429

37 Zhang H, Xu X D, Li J Y, et al. Multicell power allocation method based on game theory for inter-cell interference coordination. Sci China Ser F-Inf Sci, 2009, 52: 2378-2384

38 3GPP TR 36.912. Feasibility study for further advancements for E-UTRA (LTE-Advanced). Technical Report TR 36.912 v9.3.0, 2010

39 3GPP TR 36.808. Evolved Universal Terrestrial Radio Access (E-UTRA); Carrier aggregation base station (BS) radio transmission and reception. Technical Report TR 36.808 v1.0.0, 2010

40 3GPP TR 36.814. Further advancements for E-UTRA. Technical Report TR 36.814, v 9.0.0, 2010

41 Yuan G X, Zhang X, Wang W B, et al. Carrier aggregation for LTEadvanced mobile communication systems. IEEE Commun Mag, 2010, 48: 88-93

42 Chen L, Chen W W, Zhang X, et al. Analysis and simulation for 
spectrum aggregation in LTE-Advanced system. In: Proceedings of the 70th IEEE Vehicular Technology Conference, 2009 Sept 20-23, Anchorage. Washington DC: IEEE, 2009. 1-6

43 Zhang L, Liu F, Huang L, et al. Traffic load balance methods in the LTE-Advanced system with carrier aggregation. In: Proceedings of International Conference on Communications, Circuits and Systems, 2010 July 28-30, Chengdu. Washington DC: IEEE, 2010. 63-67

44 Meucci F, Cabral O, Velez F J, et al. Spectrum aggregation with multi-band user allocation over two frequency bands. In: Proceedings of IEEE Mobile WiMAX Symposium, 2009 July 9-10, Napa Valley. Washington DC: IEEE, 2009. 81-86

45 Zhang L, Wang Y Y, Huang L. QoS performance analysis on carrier aggregation based LTE-A systems. In: Proceedings of IET International Communication Conference on Wireless Mobile and Computing, 2009 Dec 7-9, Shanghai. New York: Curran Associates, 2009. 253-256

46 Bian H L, Guo C L, Feng C Y. An ICSGC algorithm for carrier assignment in downlink coordinated multi-point with carrier aggregation. In: Proceedings of the 2nd IEEE International Conference on Network Infrastructure and Digital Content, 2010 Sept 24-26, Beijing. Washington DC: IEEE, 2010. 934-938

47 Li J, Liu Y, Duan J, et al. Flexible carrier aggregation for home base station in IMT-advanced system. In: Proceedings of the 5th International Conference on Wireless Communications, Networking and Mobile Computing, 2009 Sept 24-26, Beijing. Washington DC: IEEE, 2009. 1-4

48 Yang Y, Hu H L, Xu J, et al. Relay technologies for WiMAX and LTE-Advanced mobile systems. IEEE Commun Mag, 2009, 47: 100-105

49 Timus B, Soldati P, Zander J. Implications of fairness criteria on the techno-economic viability of relaying networks. In: Proceedings of the 69th IEEE Vehicular Technology Conference, 2009 Apr 26-29, Barcelona. Washington DC: IEEE, 2009. 1-5

50 Zhao R, Li C G, Wang H R, et al. Performance analysis of fixed gain relaying systems in Nakagami-m fading channels. Chinese J Electron, 2011, 39: 162-167

$51 \mathrm{Ng} \mathrm{S} \mathrm{C}$, Zhang W X, Zhang Y, et al. Analysis of access and connectivity probabilities in vehicular relay networks. IEEE J Sel Area Commun, 2011, 29: 140-150

52 Lin W, Wu G, Zhang L, et al. Performance analysis of cooperative networks with random decode-and-forward relaying. In: Proceedings of the 10th IEEE International Conference on High Performance Computing and Communications, 2008 Sept 25-27, Dalian. Washington DC: IEEE, 2008. 526-531

53 Cheng Y S, Jiang Y X, You X H. Preamble design and synchronization algorithm for noise ratios cooperative relay systems. In: Proceedings of the 70th IEEE Vehicular Technology Conference, 2009 Sept 20-23, Anchorage. Washington DC: IEEE, 2009. 1-5

54 Hu F, Zhang H, You X H, et al. Low complexity channel estimation for novel bi-directional relaying schemes. In: Proceedings of the 69th IEEE Vehicular Technology Conference, 2009 Apr 26-29, Barcelona. Washington DC: IEEE, 2009. 1-5

55 Li Y, Zhang R F, Wang C G, et al. A distributed relay selection algorithm for cognitive radio ad-hoc networks. In: Proceedings of the 6th International Conference on Network and Service Management, 2010 Oct 25-29, Niagara Falls. Washington DC: IEEE, 2010. 439-442

56 Jiang $\mathrm{F}$, Tian $\mathrm{H}$, Zhang P. An adaptive random access strategy for multi-channel relaying networks. Sci China Ser F-Inf Sci, 2009, 52: 2406-2414

57 Zhang G P, Yang K, Ding E J. Power allocation scheme for selfish cooperative communications based on game theory and particle swarm optimizer. Sci China Inf Sci, 2010, 53: 1908-1912

$58 \mathrm{Li} \mathrm{Y}$, Wang Y, Zhao W L, et al. A fast handover scheme in IEEE802.16 relay networks. In: Proceedings of the 2nd International Conference on Future Networks, 2010 Jan 22-24, Sanya. Washington DC: IEEE, 2010. 243-247

59 Cao L, Yang D C, Yang H W, et al. Asymptotic performance of amplify-and-forward MIMO relaying with transmit antenna selection.
Sci China Inf Sci, 2010, 53: 2631-2641

60 Zhao R, Yang L X, Zhu W P. Transmission scheme and performance analysis for decode-and-forward MIMO two-way relay systems. Sci China Ser F-Inf Sci, 2009, 52: 2308-2316

61 Zhou M U, Li L H, Wang H F, et al. Optimal power allocation for MIMO-OFDM relaying systems. Chin J Electron, 2009, 37: 26-30

62 Fu Y H, Yang L X, Zhu W P. Optimal amplify-and-forward relaying schemes for two-hop MIMO relay networks. Chin J Electron, 2010, 19: 717-722

$63 \mathrm{Xu} \mathrm{X} \mathrm{R,} \mathrm{Zheng} \mathrm{B} \mathrm{Y.} \mathrm{Novel} \mathrm{multi-relay} \mathrm{cross-layer} \mathrm{cooperative}$ communication strategy based on Jackson queuing model. Sci China Inf Sci, 2010, 53: 842-853

64 Chen Y, Yang Y, Darwazeh I. A cross-layer analytical model of end-to-end delay performance for wireless multi-hop environments. In: Proceedings of IEEE Globecom 2010 Conference, 2010 Dec 6-10, Miami. Washington DC: IEEE, 2010. 5-10

65 Yucek T H, Arslan H. A survey of spectrum sensing algorithms for cognitive radio applications. IEEE Commun Surveys Tutorials, 2009, 11: $116-130$

66 Zhu J, Zheng B Y, Zou Y L. Detection time analysis for the multiple-user cooperative spectrum sensing scheme in cognitive radio networks. Sci China Ser F-Inf Sci, 2009, 52: 1915-1925

$67 \mathrm{Xu} \mathrm{F} \mathrm{M,} \mathrm{Zheng} \mathrm{X} \mathrm{F,} \mathrm{Li} \mathrm{X} \mathrm{Q,} \mathrm{et} \mathrm{al.} \mathrm{Partial} \mathrm{cooperative} \mathrm{spectrum}$ sensing schedule in cognitive network. Sci China Ser F-Inf Sci, 2009, 52: 2332-2341

68 Yue W J, Zheng B Y, Meng Q M, et al. Robust cooperative spectrum sensing schemes for fading channels in cognitive radio networks. Sci China Inf Sci, 2011, 54: 348-359

69 Yuan G X, Grammenos R C, Yang Y, et al. Performance analysis of selective opportunistic spectrum access with traffic prediction. IEEE Trans Veh Technol, 2010, 59: 1949-1959

70 Sun Y Z, Hu H L, Liu F Q, et al. Dynamic spectrum access based on MAC-layer spectrum sensing and prior channel pre-allocation strategy. IEICE Trans Commun, 2010, E93.B: 609-619

71 Che Y L, Chen J, Tang W B, et al. A two-step channel and power allocation scheme in centralized cognitive networks based on fairness. In: Proceedings of the 67th IEEE Vehicular Technology Conference, 2008 May 11-14, Marina Bay. Washington DC: IEEE, 2008, 1589-1593

72 Guo K Q, Sun L X, Li Y, et al. Channel-aware and queue-aware joint-layer resource optimization for cognitive radio networks. Sci China Inf Sci, 2010, 53: 2576-2583

73 Rong M, Zhu S H, Li F. Spectrum sharing based on Frobenius norm in cognitive radio networks. Chin J Electron, 2011, 39: 95-100

74 Wang P, Chen X, Zhou S D, et al. On ergodic capacity of spectrum-sharing systems in fading channels. IEICE Trans Commun, 2009, E92.B: 1904-1907

75 Han C, Wang J, Yang Y L, et al. Addressing the control channel design problem: OFDM-based transform domain communication system in cognitive radio. Comput Netw, 2008, 52: 795-815

76 Le V B, Lin Y W, Wang X M, et al. A cell based dynamic spectrum management scheme with interference mitigation for cognitive networks. In: Proceedings of the 67th IEEE Vehicular Technology Conference, 2008 May 11-14, Marina Bay. Washington DC: IEEE, 2008, 1594-1598

77 Wang P, Zhao M, Xiao L M, et al. Power allocation in OFDM-based cognitive radio systems. In: Proceedings of IEEE Globecom 2007 Conference, 2007 Nov 26-30, Washington DC. Washington DC: IEEE, 2010. 4061-4065

$78 \mathrm{Xi} \mathrm{W}$, Yin C, Yue G. Joint beamforming and scheduling in the downlink of cognitive radio networks. Sci China Ser F-Inf Sci, 2009, 52: 2324-2331

79 Yue W J, Zheng B Y, Meng Q M, et al. Optimal power allocation for cognitive relay networks: Amplify-and-forward versus selection relay. Sci China Inf Sci, 2011, 54: 861-872

80 Hu H L, Zhang Y, Luo J J. Distributed Antenna Systems: Open Architecture for Future Wireless Communications. Boca Raton: CRC Press, 2007

81 Hu X W, Zhang Y, Jia Y Z, et al. Power coverage and fading char- 
acteristics of indoor distributed antenna systems. In: Proceedings of the 4th International Conference on Communications and Networking in China, 2009 Aug 26-28, Xian. Washington DC: IEEE, 2009. 1066-1069

82 Feng W, Li Y Z, Zhou S D, et al. Downlink capacity of distributed antenna systems in a multi-cell environment. In: Proceedings of IEEE Wireless Communications \& Networking Conference, 2009 Apr 5-8, Budapest. Washington DC: IEEE, 2009. 847-851

83 Chen H M, Chen M. Capacity of the distributed antenna systems over shadowed fading channels. In: Proceedings of the 69th IEEE Vehicular Technology Conference, 2009 Apr 26-29, Barcelona. Washington DC: IEEE, 2009. 1-5

84 Hu H L, Weckerle M, Luo J. Adaptive transmission mode selection scheme for distributed wireless communication systems. IEEE Commun Lett, 2006, 10: 573-575

85 Zhao X S, You X H, Zhu D Q, et al. A capacity-based RAU selection scheme for downlink transmission in distributed antenna system. In: Proceedings of International Conference on Wireless Communications and Mobile Computing, 2006 July 3-6, Vancouver. Washington DC: IEEE, 2006. 485-490

86 Zhang N B, Kang G X, Guo Y Y, et al. Symbol error rate analysis and antenna selection in limited feedback distributed antenna systems. In: Proceedings of the 72nd IEEE Vehicular Technology Conference, 2010 Sept 6-9, Ottawa. Washington DC: IEEE, 2010. 1-5

87 Feng W, Zhang X J, Zhou S D, et al. Downlink power allocation for distributed antenna systems with random antenna layout. In: Proceedings of the 69th IEEE Vehicular Technology Conference, 2009 Apr 26-29, Barcelona. Washington DC: IEEE, 2009. 1-5

88 Wu T, Kwon Y H, Zhang J Y, et al. Distributed antenna systems with power adjusted beam switching. In: Proceedings of the 71st IEEE Vehicular Technology Conference, 2010 May 16-19, Taipei. Washington DC: IEEE, 2010. 1-5

89 Wang X Z, Zhu P C, Chen M. Antenna location design for generalized distributed antenna systems. IEEE Commun Lett, 2009, 13: $315-317$

90 Jiang Z J, Wu J, Wang D M, et al. Parallel proportion fair scheduling in DAS with partial channel state information. IEICE Trans Commun, 2009, E92.B: 2312-2315

91 You X H, Wang D M, Sheng B, et al. Cooperative distributed antenna systems for mobile communications. IEEE Wirel Commun, 2010, 17: 35-43

92 Deng K, Tang Y X , Lei X, et al. Estimation of carrier frequency offsets for MIMO systems with distributed transmit antennas. J Electron (China), 2007, 24: 455-461

93 Wang D M, You X H, Wang J Z, et al. Spectral efficiency of distributed MIMO cellular systems in a composite fading channel. In: Proceedings of International Conference on Communications, 2008 May 19-23, Beijing. Washington DC: IEEE, 2008. 1259-1264

94 Wang Y, Sun K, Chen Z X. Comparison of downlink transmission strategies for distributed antenna systems with multi-antenna arrays. J China Univ Posts Telecommun, 2010, 17: 44-50

95 Zhang N B, Kang G X, Guo Y Y, et al. A low-complexity port selection and power allocation scheme in distribution MIMO systems. J Electron (China), 2010, 27: 145-150

96 Choi J. 4G, Solution for convergence? IEEE MTT-S International Microwave Symposium Digest, 2006. 843-846
97 Perkins C. IP Mobility Support. IETF RFC 2002, 1996

98 Marchese M. QoS Over Heterogeneous Networks. Chichester: John Wiley \& Sons, 2007

99 Narang N, Mittal R. Network management for next generation networks. In: Proceedings of the 8th International Conference on Advanced Computing and Communications, 2000 Dec 14-16, Cochin. New York: Curran Associates, 2000. 1-4

$100 \mathrm{Hu}$ Y X, Lan J L, Wu J X. Providing personalized converged services based on flexible network reconfiguration. Sci China Inf Sci, 2011, 54: 334-347

101 3GPP TR 36.902. Self-configuring and self-optimizing network use cases and solutions. Technical Report TR 36.902 v9.3.0, 2010

102 Hu H L, Zhang J, Zheng X Y, et al. Self-configuration and self-optimization for LTE networks. IEEE Commun Mag, 2010, 48: 94-100

103 Feng Z Y, Liang L T, Tan L, et al. Q-learning based heterogeneous network self-optimization for reconfigurable network with CPC assistance. Sci China Ser F-Inf Sci, 2009, 52: 2360-2368

104 Yilmaz O. Self-optimization of coverage and capacity in LTE using adaptive antenna systems. Master's Thesis, Helsinki: Aalto University, 2010

105 Jansen T, Balan I, Turk J, et al. Handover parameter optimization in LTE self-organizing networks. In: Proceedings of the 72nd IEEE Vehicular Technology Conference, 2010 Sept 6-9, Ottawa. Washington DC: IEEE, 2010. 1-5

106 Zhang H J, Wen X M, Wang B, et al. A novel self-optimizing handover mechanism for multi-service provisioning in LTE-Advanced. In: Proceedings of International Conference on Research Challenges in Computer Science, 2009 Dec 28-29, Shanghai. Washington DC: IEEE, 2009. 221-224

107 Zhou Y, Yuan J, Wang Y, et al. Service-oriented FMIPv6 framework for efficient handovers in $4 \mathrm{G}$ networks. In: Proceedings of IEEE Globecom 2007 Conference, 2007 Nov 26-30, Washington DC. Washington DC: IEEE, 2010. 4478-4482

108 Liu X, Jiang L, He C. Performance evaluations of an IDMP-based macro-mobility management in heterogeneous wireless networks. Chinese J Electron, 2009, 18: 530-534

109 Lobinger A, Stefanski S, Jansen T, et al. Load balancing in downlink LTE self-optimizing networks. In: Proceedings of the 71st IEEE Vehicular Technology Conference, 2010 May 16-19, Taipei. Washington DC: IEEE, 2010. 1-5

110 Wang H, Ding L H, Wu P, et al. Dynamic load balancing and throughput optimization in 3GPP LTE networks. In: Proceedings of the International Conference on Wireless Communications and Mobile Computing, 2010 June 28-July 2, Caen. Washington DC: IEEE, 2010. 939-943

111 Xu H B, Tian H, Huang B, et al. An improved dynamic user equipment power saving mechanism for LTE system and performance analysis. Sci China Inf Sci, 2010, 53: 2075-2086

$112 \mathrm{Xu} \mathrm{J}$, Liu C, Yang Y, et al. An overview of energy efficiency analytical models in communications networks. In: Proceedings of International Conference on Wireless Communications and Signal Processing, 2010 Oct 21-23, Suzhou. Washington DC: IEEE, 2010. $21-23$

113 You X H, Wang J, Zhang P, et al. Study and ideas for green wireless mobile communications. J Univ Sci Technol China, 2009, 39: 1009-1015

Open Access This article is distributed under the terms of the Creative Commons Attribution License which permits any use, distribution, and reproduction in any medium, provided the original author(s) and source are credited. 